\title{
Effects of estrogen deficiency combined with chronic alcohol consumption on rat mandibular condyle
}

\author{
Miriane Carneiro Machado Salgado ${ }^{1}$, Adriana Mathias Pereira da Silva Marchini ${ }^{1}$, Tábata de Mello Tera ${ }^{1}$, \\ Rosilene Fernandes da Rocha ${ }^{1}$, Leonardo Marchini ${ }^{2}$
}

${ }^{1}$ Universidade Estadual Paulista - UNESP, Instituto de Ciência e Tecnologia, Department of Dentistry, São José dos Campos, SP, Brazil

${ }^{2}$ University of lowa, College of Dentistry, Department of Preventive and Community Dentistry, lowa City, lowa, United States of America

Abstract

\begin{abstract}
Estrogen deficiency and chronic alcohol consumption may have a synergistic and deleterious effect on bone tissue. Aim: To investigate the effects of estrogen deficiency associated with chronic alcohol consumption on the mandibular condyle in rats. Methods: Fifty-four female rats were first divided equally into two groups: ovariectomized (Ovx) and simulated ovariectomy (Sham). One month after the surgeries, these groups were equally sub-divided according to their dietary treatment: G1: Sham/ad-libitum diet; G2: Sham/alcohol; G3: Sham/isocaloric; G4: Ovx/ad-libitum diet; G5: Ovx/alcohol, G6: Ovx/isocaloric. Eight weeks after starting the diets, all animals were anesthetized and sacrificed. The condyles were analyzed histologically, histomorphometrically, and immunohistochemically using the antibodies for bone sialoprotein $(B S P)$, osteocalcin (OCC) and receptor activator of nuclear factor kappa-B ligand (RANKL). Results: Histological analysis of the mandibular condyles showed that Ovx and Sham groups presented almost the same characteristics. The histomorphometric analysis showed that there was a statistically significant difference only between Ovx/isocaloric and Ovx/ad-libitum groups $(\mathrm{p}=0.049)$. No difference was observed in the intensity of BSP, OCC, and RANKL antibody staining between the Ovx/alcohol and the other groups. Conclusions: It may be concluded that there was no histomorphometric, histological, or RANKL, BSP, and OCC staining differences between the Ovx/alcohol group and other experimental groups.
\end{abstract}

Keywords: mandible; mandibular condyle; ethanol; ovariectomy.

\section{Introduction}

Estrogen is a steroid hormone that is important for the growth and maintenance of the female skeleton and inhibits bone resorption ${ }^{1}$. Estrogen deficiency is associated with increased bone resorption relative to bone formation, causing excessive loss of bone mineral density. Bone resorption increases due to increased osteoclastogenesis and decreased apoptosis of osteoclasts ${ }^{2}$. These events culminate in the imbalance of bone remodeling, which can contribute to the occurrence of osteoporosis ${ }^{3}$.

Osteoporosis is a chronic and progressive skeletal disorder related to bone metabolism in which the bones become less resistant to fractures. The amount of bone tissue is reduced, the bone presents larger resorption areas and bone surface becomes irregular. Thus, there is skeletal fragility and, consequently, an increased 
risk of fractures ${ }^{4}$. With the growing elderly population, more people may be likely to develop osteoporosis and consequently suffer from fractures. Considering that fractures lead to high-cost treatment, osteoporosis is considered a public health problem ${ }^{3}$.

The effects of osteoporosis are greater in long bones, such as the femur and radius, and also in vertebrae ${ }^{5}$. However, there is evidence that osteoporosis also occurs in the jaw ${ }^{4}$. The relationship between systemic bone loss and bone loss in the jaw for patients with osteoporosis has been reported in the literature ${ }^{6}$.

Bone metabolism in the mandibular condyle is heavily influenced by sex hormones, especially estrogen, suggesting that the absence of estrogen may influence condyle bone remodeling and potentially lead to degenerative changes in the temporomandibular joint ${ }^{7}$. Studies have shown that ovariectomy induces bone loss in the whole mandible, including its condyle ${ }^{7-11}$. Structural defects in mandibular condyles were found in patients diagnosed with severe osteoporosis after menopause, indicating that osteoporosis can change mandibular condyle structure ${ }^{12}$.

Alcohol consumption inhibits bone formation by decreasing osteoblast count and proliferation. However, the decrease in bone formation and increase in resorption may indicate that alcohol not only inhibits osteoblastic activity, but also stimulates osteoclastic activity. Excessive alcohol consumption results in progressive bone loss and increased risk for osteoporosis development and, consequently, increased risk of osteoporosis-related fractures ${ }^{13,14}$.

In the orofacial region, the bone most commonly affected by fractures is the mandible ${ }^{15}$. Two-thirds of the fractures of the maxillofacial region occur in the mandible, and the condyle region is the most commonly fractured mandibular site, followed by the symphysis, body, alveolar region, angle, and ramus ${ }^{16}$.

Thus, considering 1) estrogen deficiency and its relationship with osteoporosis; 2) osteoporosis and its implications for bone fragility; 3) alcohol consumption and its consequences for bone tissue; 4) the high incidence of condyle fractures among orofacial fractures; and 5) the absence of studies that investigate the influence of osteoporosis in conjunction with alcohol consumption in the mandibular condyle region, this study aims to investigate the effects of estrogen deficiency combined with chronic alcohol consumption on the mandibular condyle of rats. In this way, the hypothesis to be tested is that estrogen deficiency combined with chronic alcohol consumption might have deleterious effect on the bone structure of the mandibular condyle in rats.

\section{Material and methods}

\section{Animals}

Fifty-four female rats (Rattus norvegicus albinus, variation Wistar) were used. The animals were three months of age in the beginning of the experiment, and the study was carried out according to the ethical principles established by the Brazilian College of Experiments Involving Animals
(COBEA in Portuguese) and approved by the Ethics Committee for Research Involving Animals of the State University of São Paulo-UNESP, under the Protocol \#0122011-PACEP.

The animals were first equally divided into two groups: ovariectomized (Ovx) and simulated ovariectomy (Sham). One month after surgeries, these groups were equally subdivided according to the dietary treatment: G1: Sham/adlibitum diet; G2: Sham/alcohol; G3: Sham/isocaloric; G4: Ovx/ad-libitum diet; G5: Ovx/alcohol, G6: Ovx/isocaloric. Therefore, the animals were divided into six experimental groups with nine $(n=9)$ animals in each group (totalizing 54 animals in the whole experiment).

The solid food offered to all groups was a commercial rat food (Labina, Purina of Brazil, Paulínia-Brazil). For G2 and $\mathrm{G} 5$, that received alcohol, 50 grams of food were provided daily to each animal. The next day, the remaining food was weighed and averaged, and the average quantity of food was provided to each animal in G3 and G6, the isocaloric groups. G1 and G4 received ad-libitum solid food.

Animals from groups G2 and G5 received 20\% alcohol solution obtained by diluting absolute ethanol (ABS.ACS99.5 $5^{\circ}$ Ecibra, São Paulo, SP, Brazil) in purified water. Initially, these animals were submitted to a nine-day adaptation period: three days receiving a $5 \%$ alcohol solution followed by three days receiving $10 \%$ alcohol solution, and then three days receiving $15 \%$ alcohol solution. Subsequently, they received the prepared $20 \%$ alcoholic solution daily for eight weeks.

G2 and G5 groups (the alcohol groups) began one day before G3 and G6 (the isocaloric groups), and they were provided $50 \mathrm{~mL}$ of alcohol solution per animal. The next day, the remaining solution was measured and the intake averaged. The isocaloric solution was then prepared by dissolving $266 \mathrm{~g}$ of sucrose into $1 \mathrm{~L}$ of water. The calculations for the quantity of isocaloric solution to be offered to G3 and G6 were made by taking into consideration the alcohol concentration used in this study (20\%), the density of absolute alcohol $(0.787 \mathrm{~g} / \mathrm{mL})$, the caloric values of sucrose $(4.1 \mathrm{kcal} /$ g) and alcohol $(7.1 \mathrm{kcal} / \mathrm{g})$. In addition to the isocaloric solution, groups G3 and G6 also received water ad-libitum.

The average values for solid and liquid consumption per cage were recorded daily. From these data, the average consumption per animal was calculated for each experimental group as food intake ( $\mathrm{g}$ and $\mathrm{Kcal}$ ), liquid solution $(\mathrm{mL}$ and $\mathrm{Kcal}$ ), total calories (Kcal), and percentage of calories from liquid and solid food.

\section{Anesthesia}

The rats were anesthetized by intramuscular injection of $0.1 \mathrm{~mL} / \mathrm{kg}$ of 1.25:1 mixture of xylazine chloride (Anasedan; Vetbrands, Jacareí, São Paulo, SP, Brazil) and ketamine chloride (Dopalen; Vetbrands) whenever necessary during the procedures described below.

\section{Ovariectomy and Sham surgery}

The rats belonging to Ovx groups (G4, G5, and G6) were ovariectomized at three months of age. Animals from 
Sham groups (G1, G2, and G3) had their ovaries exposed and immediately reseated in the abdominal cavity at the same procedure to simulate the surgical stress of an ovariectomy. After the surgery, all rats were given a single dose of intramuscular antibiotics (benzathine benzylpenicillin, procaine benzylpenicillin, benzylpenicillin potassium, and dihydrostreptomycin sulphate [Pentabiótico; Fort Dodge Saúde Animal, Campinas, SP, Brazil, $1.35 \mathrm{~mL} / \mathrm{kg}]$ ) and an anti-inflammatory (diclofenac sodium $75 \mathrm{mg}$ [Voltaren injetável; Ciba-Geigy, Rio de Janeiro, RJ, Brazil, 1 mg/kg]).

\section{Euthanasia}

Eight weeks after starting diets, all animals were anesthetized and sacrificed. All animals were weighed at the beginning of the experiment and immediately prior to sacrifice.

\section{Histological and immunohistochemical analysis}

The mandibles were cleaned and placed in $10 \%$ formaldehyde solution for $48 \mathrm{~h}$. Then, the samples were decalcified in EDTA solution and subsequently embedded in paraffin. Histological sections were performed in the frontal plane of the condyle. $3-\mu \mathrm{m}$ sections were mounted on silanized slides for immunohistochemical procedures, and 4- $\mu \mathrm{m}$ sections were used for hematoxylin-eosin staining.

The primary antibodies used in this study were those for bone sialoprotein (BSP-LF-87), osteocalcin (OCC - FL110: sc-30044), and receptor activator of nuclear factor kappaB ligand (RANKL - N-19: sc-7628). Their titration, time and temperature of incubation, and antigen recovery are listed in Table 1. Samples were incubated with a biotinylated secondary antibody for $30 \mathrm{~min}$ and exposed to a streptavidin"peroxidase tertiary complex, also for $30 \mathrm{~min}$ (Universal Dako LSAB ${ }^{\circledR}$ Kit, Peroxidase, Carpinteria, CA, USA). They were then incubated with diaminobenzidine solution for 2 min (Dako Liquid DAB). Sections were counterstained with Mayer's hematoxylin. Negative controls were achieved using an equivalent solution for primary antibody dilution, but without the antibody itself. Positive controls were made according to the instructions of primary antibody manufacturers.

The images of histological and immunohistochemical slides were photographed by a high-resolution digital camera (AxioCam MRc5; Carl Zeiss, Oberköchen, Germany) coupled to a microscope (Axiophot 2; Carl Zeiss). The camera is connected to a computer containing image acquisition and analysis software (Axiovision Release 4.7.2).

In the immunohistochemical analysis, the following cells were analyzed: osteoblasts, osteoclasts, chondrocytes and

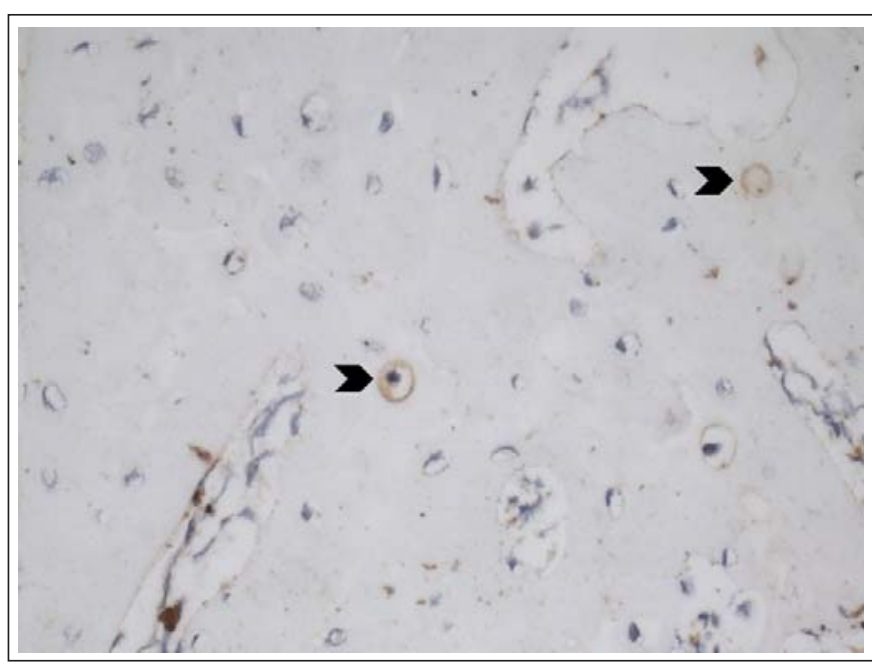

Fig.1 - Was considered staining positive for osteocyte when the osteocyte lacunae was stained (arrows) - Antibody - RANKL.

osteocytes. Staining was considered positive for osteocyte when the osteocyte lacunae were stained (Figure 1).

For all groups, cell types and antibodies, the staining intensity was classified either as weak, moderate or intense ${ }^{17}$. The staining analysis took into account both degree of color (lighter colors were considered weaker) and degree of heterogeneity (more heterogeneous patterns were considered weaker). For the cases were analysis was challenging, the cells were visualized in higher magnification.

\section{Histomorphometric analysis}

The Image-J software was used for histomorphometric analysis of trabecular bone by counting points in a grid. Only the points that fell on trabecular bone were counted, excluding points on osteocytes, medullary spaces, osteoblasts, osteoclasts, and bone marrow cells.

\section{Data analysis}

Weight changes and variations in food and liquid intake were subjected to Kruskal-Wallis and Mann-Whitney tests. The data obtained from the histomorphometric analysis were subjected to Anova and Tukey variance tests. All tests used a significance level of 5\%. Histological and immunohistochemical data were analyzed qualitatively.

\section{Results}

There was an average weight gain in all groups. The group that gained the most weight was the Ovx/ad-libitum group (average gain of $40.83 \% \pm 8.59 \%$ ), which gained

Table 1 - Antibody, titration, time and temperature of incubation, and antigenic recovery.

\begin{tabular}{lccc}
\hline Antibody & Titration & Incubation & Antigenic recovery \\
Bone sialoprotein (BSP) & $1: 100$ & Room temperature, 1h & Citrate \\
Osteocalcin (OCC) & $1: 100$ & $4{ }^{\circ} \mathrm{C}$, overnight & Citrate \\
Receptor activator of nuclear & $1: 75$ & $4{ }^{\circ} \mathrm{C}$, overnight & Pepsin \\
factor kappa-B ligand (RANKL) & & & \\
\hline
\end{tabular}


Table 2 - Comparison among experimental groups regarding trabecular bone area (in percentage [\%] of the total area).

\begin{tabular}{lcccccc}
\hline & Ovx/alcohol & Ovxlad-libitum & Ovxlisocaloric & Sham/alcohol & Sham/ ad-libitum & Sham/isocaloric \\
Average & 57.83 & 55.42 & 65.10 & 59.72 & 56.09 & 63.29 \\
Median & 57.81 & 57.29 & 66.14 & 59.89 & 54.68 & 63.54 \\
SD & 12.79 & 12.81 & 11.18 & 12.76 & 14.00 & 9.53 \\
Min & 36.45 & 30.72 & 40.10 & 32.29 & 32.39 & 37.50 \\
Max & 80.20 & 76.04 & 86.45 & 88.54 & 86.45 & 80.72 \\
N & 27 & 29 & 25 & 27 & 29 & 29 \\
\hline P-value & & \multicolumn{5}{c}{$0.019^{*}$}
\end{tabular}

The asterisk (*) indicates a statistically significant difference.

$\mathrm{N}=$ number of slides used in the histological analysis in each experimental group.

significantly more weight than the other groups according to the Kruskal Wallis and Mann Whitney tests. The group that gained the least weight was the Sham/isocaloric group (average gain of $4.10 \% \pm 7.25 \%$ ), also statistically different from the others except the Sham/alcohol and Ovx/alcohol groups.

The group that consumed the most food was the Ovx/ ad-libitum group (19.26 $\mathrm{g} \pm 4.05)$, statistically different from the other groups with the exception of the Sham/ad-libitum group (Kruskal-Wallis and Mann-Whitney tests). Average food intake by the isocaloric groups was similar to the average from the alcoholic groups, indicating that isocaloric groups consumed all the food offered to them. Regarding liquid intake, animals from isocaloric groups did not consume all of the solution offered to them. The animals that were given alcohol ingested the equivalent of $8.43 \mathrm{~g}$ of absolute alcohol per $\mathrm{kg}$ per day; an average of $44.5 \%$ of their caloric intake came from alcohol.

Histological analysis of the mandibular condyles showed that Ovx and Sham groups presented almost the same characteristics. Osteoclasts were present in all condyles from the Sham/alcohol and Sham/ad-libitum groups. Only three condyles from Sham/isocaloric group presented osteoclasts, Ovx/isocaloric group presented osteoclasts in two condyles, Ovx/ad-libitum in four, and Ovx/alcohol in three. Osteoblasts were present in all groups.

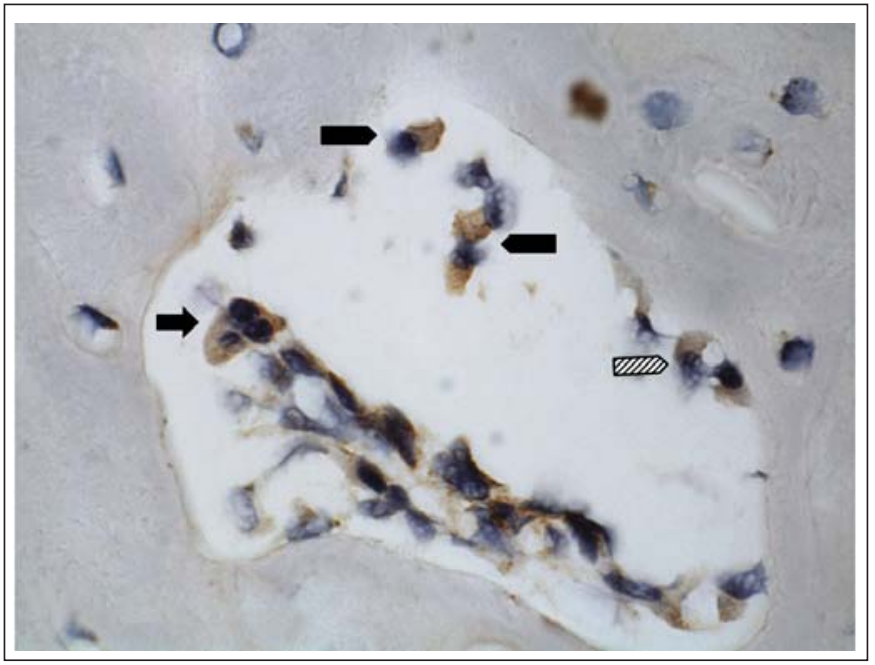

Fig. 2 - RANKL staining intensity. Intense osteoclast staining osteoblast staining ( and moderate osteoblast staining ( VIIIII
The histomorphometric analysis showed that there was a statistically significant difference (ANOVA) among the groups, as can be observed in Table 2. Using the Tukey's multiple comparison test, there was a statistically significant difference only between Ovx/isocaloric and Ovx/ad-libitum groups $(\mathrm{p}=0.049)$.

Immunohistochemical analysis showed that RANKL is expressed by osteoclasts, osteoblasts, osteocytes and chondrocytes. All experimental groups showed variations of staining intensities between weak, moderate and intense (Figure 2). The pattern of these variations can be observed in Table 3. The bone sialoprotein (BSP) staining presented variations in intensity, which varied according to the group and cell type, as shown in Table 4. BSP reacted positively on osteoblasts (Figure 3), osteocytes and chondrocytes. Osteoclast staining occurred only in Sham groups. The Sham/ alcohol group presented all three staining intensities, the Sham/ad libitum group presented weak and moderate osteoclast staining and the Sham/isocaloric group presented only moderate osteoclast staining. Osteocalcin (OCC) was expressed by osteoblasts (Figure 4), osteocytes and chondrocytes. OCC staining also presented variations of intensity, which varied according to the group and cell type, as shown in Table 5 .

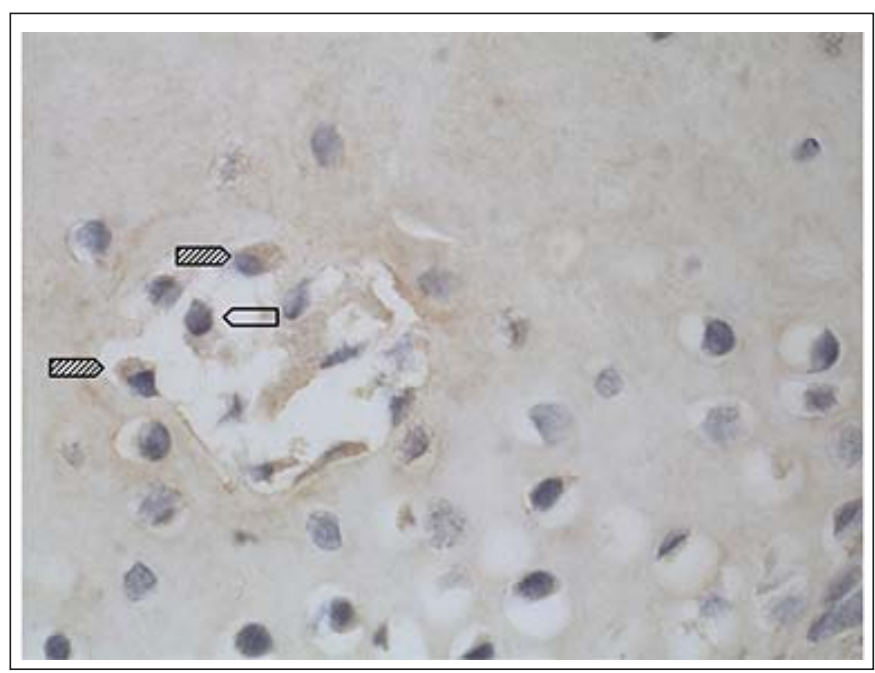

Fig. 3 - BSP staining intensity. Weak ( $\square$ ) and moderate ( staining. 
Table 3 - Receptor activator of nuclear factor kappa-B ligand (RANKL) staining intensity.

\begin{tabular}{llccc}
\hline & Osteoblasts & Osteoclasts & Osteocytes & Condrocytes \\
Sham/ alcohol & Moderate & Weak & Intense & Weak \\
Sham/ isocaloric & Intense & Weak & Moderate & Weak \\
Sham/ ad-libitum & Intense & Moderate & Weak & Moderate \\
Ovx/Alcohol & Moderate & Intense & Weak & Moderate \\
Ovx/ isocaloric & Moderate & Weak & Weak & Weak \\
Ovx/ad-libitum & Moderate & Intense & Moderate & Weak \\
\hline
\end{tabular}

Table 4 - Bone sialoprotein (BSP) staining intensity.

\begin{tabular}{|c|c|c|c|c|}
\hline & Osteoblasts & Osteoclasts & Osteocytes & Condrocytes \\
\hline Sham/ alcohol & Moderate & Weak & Weak & Moderate \\
\hline Sham/ isocaloric & Moderate & Moderate & Weak & Weak \\
\hline Sham/ ad-libitum & Weak & Weak & Weak & Intense \\
\hline Ovx/Alcohol & Moderate & - & Weak & Weak \\
\hline Ovx/ isocaloric & Intense & - & Weak & - \\
\hline Ovx/ad-libitum & Intense & - & Weak & Moderate \\
\hline
\end{tabular}

Table 5 - Osteocalcin (OCC) staining intensity.

\begin{tabular}{lcccc}
\hline & Osteoblasts & Osteoclasts & Osteocytes & Condrocytes \\
Sham/ alcohol & Weak & - & Weak & Weak \\
Sham/ isocaloric & Weak & - & Weak & Weak \\
Sham/ ad-libitum & Weak & - & Weak & Weak \\
Ovx/Alcohol & Weak & - & Weak & Weak \\
Ovx/ isocaloric & Weak & - & Weak & Weak \\
Ovx/ad-libitum & Moderate & - & Weak & Weak \\
\hline
\end{tabular}

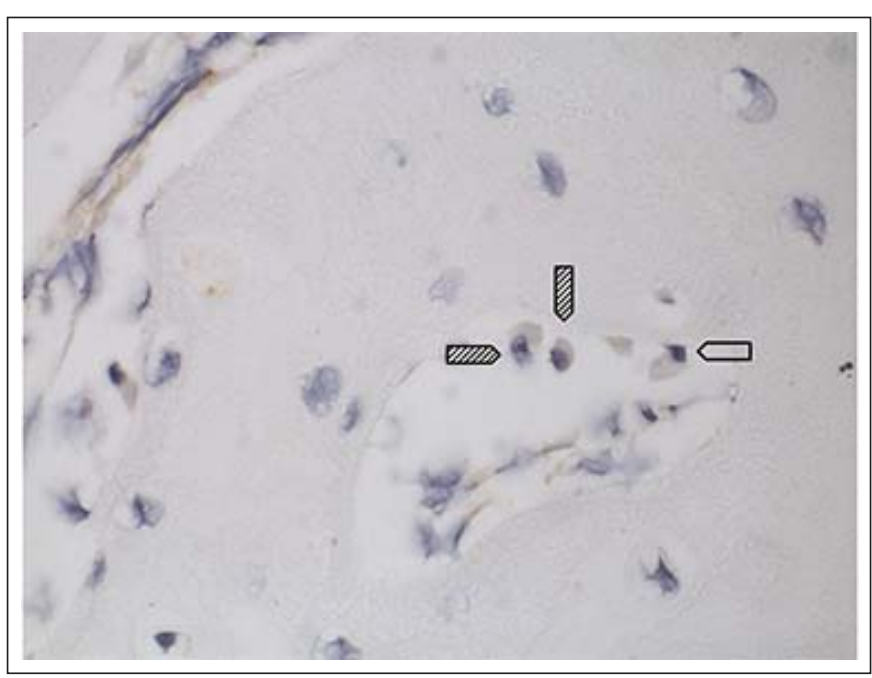

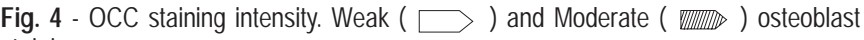
staining

\section{Discussion}

In the present study, the average alcohol consumption of $8.43 \mathrm{~g}$ alcohol per $\mathrm{kg}$ per day suggests that the animals exhibited chronic and excessive alcohol consumption. The harmful effects of alcohol on the bone are noted in alcohol abuse, but not in moderate alcohol consumption ${ }^{18,19}$. A previous study ${ }^{20}$ observed animals consuming $7.6 \mathrm{~g}$ of $95 \%$ alcohol per kg per day, which would be equivalent to the daily consumption of $2.5 \mathrm{~L}$ beer or $1 \mathrm{~L}$ wine for an adult man. Another study ${ }^{21}$ reported that $8.76 \mathrm{~g}$ of alcohol per $\mathrm{kg}$ per day were consumed by their animals, close to the values found in this study.

In this study, an average of $44.5 \%$ of caloric intake was derived from alcohol. However, these calories from alcohol are empty, i.e. are not related to protein, vitamins or minerals, leading to nutritional deficiencies due to low nutrient intake $^{22}$. It was decided to use isocaloric groups as nutritional controls to simulate the nutrient deficiency associated with alcohol consumption ${ }^{21,23}$. However, one of the limitations of this study was that the animals of the isocaloric groups were not pushed to consume the same amount of calories from liquid, as did those of the alcohol groups. Therefore, the isocaloric groups ingested less sucrose volume compared to the alcohol groups, and so they had an even more calorierestricted diet compared to the alcohol groups. In regard to solid diet, the animals of the isocaloric groups consumed the entire amount of provided food (average of $9.39 \mathrm{~g}$ per day per animal).

Animals from all groups gained weight, which was reported previously ${ }^{24,25}$. In the present study, the Ovx/adlibitum group gained significantly more weight from all the other groups, as reported earlier ${ }^{21}$, which contradicts another study $^{25}$ that found no statistically significant differences. The Ovx/ad-libitum group also consumed more food, as reported earlier ${ }^{21}$. Ovariectomy can increase food intake and weight gain $^{26,27}$, and a study ${ }^{27}$ verified that Ovx animals showed a greater increase in body weight compared to Sham-operated 
animals. In addition, the alcohol groups ingested a much smaller amount of food than the groups that were on adlibitum diets. This was expected, since alcohol is a substance with low nutritional value and high energy content, causing the animals to feel satiety.

Histological results showed no significant difference between the Ovx/alcohol group and the other groups. Additionally, Ovx groups showed a smaller number of osteoclasts relative to Sham groups. Bone metabolism in the mandibular condyle is heavily influenced by estrogen, suggesting that the absence of estrogen influences condylar bone remodeling and may lead to degenerative changes in the temporomandibular joint ${ }^{7}$. In addition to ovariectomy increasing the number of osteoclasts ${ }^{7}$, decreased numbers of osteoclasts and osteoblasts in both Sham and Ovx groups were observed sixty days after surgeries ${ }^{28}$. However, contrary to the present results, another study found that the number of osteoclasts in Ovx animals was higher than in Sham animals ${ }^{11}$.

Histomorphometric results also revealed that the Ovx/ alcohol group was not different from other groups. Only between the Ovx/ad-libitum and Ovx/isocaloric groups there was a statistically significant difference regarding the percentage of trabecular bone. This result shows that diet seems to have an influence on the amount of mandibular condyle trabecular bone in Ovx rats, since the Ovx-ad-libitum group consumed more food. However, in Sham groups there was no difference between the ad-libitum, isocaloric or alcohol groups.

In the present study, antibodies for receptor activator of nuclear factor kappa- $\beta$ ligand (RANKL), bone sialoprotein (BSP), and osteocalcin (OCC) were used for immunohistochemical analysis. They are some of the noncollagenous proteins in the bone matrix. Non-collagenous proteins play an important role in the organization of the collagen matrix and in regulating the formation and growth of hydroxyapatite crystals ${ }^{29}$.

RANKL is a cytokine that belongs to the family of tumor necrosis factors $(\mathrm{TNF})^{30}$. It increases the activity and prolongs the lifetime of osteoclasts by decreasing apoptosis. RANKL is a mediator of the formation, function and survival of osteoclasts, and it is considered a main mediator of bone resorption ${ }^{31,32}$. BSP is produced by osteoblasts, osteoclasts, osteocytes and hypertrophic chondrocytes ${ }^{33}$. BSP is also a powerful regulator of the differentiation and activity of osteoblasts, and it has fundamental importance in the early stages of osteogenesis ${ }^{34}$. However, BSP is also recognized to induce cell adhesion, increasing the synthesis of osteoclasts and bone resorption. OCC is a small protein found exclusively in mineralized tissues ${ }^{31}$. It is considered a marker of osteoblast activity and bone formation ${ }^{13}$. OCC is expressed in the final stages of osteoblast differentiation in the bone maturation and mineralization processes ${ }^{35}$.

A previous study ${ }^{36}$ reported intense and moderate RANKL staining on osteoblasts and osteoclasts. Positive BSP staining was also previously reported ${ }^{12}$ during the first week after ovariectomy and OCC expression was clearly changed by ovariectomy ${ }^{37}$.
In this study, slides were analyzed qualitatively according to the intensity of immunohistochemistry reaction, as previously conducted by other authors ${ }^{17,38,39}$. Qualitative analysis has some limitations, since it does not provide numerical estimates and rely on subjective human observation. Furthermore, the antigen antibody reactions could be not be stoichiometric, so a strong intensity reaction (darkness of stain) does not necessarily mean greater amount of reaction products, which can be considered another limitation of this study.

Considering these effects of ovariectomy and reported deleterious effects of alcohol consumption on bone tissues ${ }^{14,21,25}$, important modifications of RANKL, BSP, and OCC staining intensities were anticipated in the Ovx/alcohol group compared to other groups. However, similar to the histological and histomorphometric results, no differences were found between the Ovx/alcohol group and other groups, thus rejecting the study hypothesis.

\section{Acknowledgements}

The authors would like to thank FAPESP for its support (São Paulo State Research Support Foundation, grant \#2011/ 03447-0). The authors also thank Ms. Anna Okulist for her native English proofreading of this manuscript.

\section{References}

1. Manolagas SC, Kousteni S, Jilka RI. Sex steroids and bone. Recent Prog Horm Res. 2002; 57: 385-409.

2. Manolagas SC. Birth and death of bone cells: basic regulatory mechanisms and implications for the pathogenesis and treatment of osteoporosis. Endocr Rev. 2000; 21: 115-37.

3. Faloni APS, Cerri PS. Cellular and molecular mechanisms of the estrogen in the bone resorption. Rev Odontol UNESP. 2007; 36: 181-8.

4. Dervis E. Oral implications of osteoporosis. Oral Surg Oral Med Oral Pathol Oral Radiol Endod. 2005; 100: 349-56.

5. Ejiri S, Tanaka M, Watanabe N, Anwar RB, Yamashita E, Yamada K, et al. Estrogen deficiency and its effect on the jaw bones. J Bone Miner Metab. 2008; 26: 409-15.

6. Jeffcoat M. The association between osteoporosis and oral bone loss. J Periodontol. 2005; 76: 2125-32.

7. Fujita T, Kawata T, Tokimasa C, Kohno S, Kaku M, Tanne K. Breadth of the mandibular condyle affected by disturbances of the sex hormones in ovariectomized and orchiectomized mice. Clin Orthod Res. 2001; 4: 172-6.

8. Fujita T, Kawata T, Tokimasa C, Kaku M, Kawasoko S, Tanne K. Influences of ovariectomy and orchiectomy on the remodeling of mandibular condyle in mice. J Craniofac Genet Dev Biol. 1998; 18: 164-70.

9. Tanaka M, Ejiri S, Kohno S, Ozawa H.. The effect of aging and ovariectomy on mandibular condyle in rats. J Prosthet Dent. 1998; 79: 685-90.

10. Nakajima M, Ejiri S, Tanaka M, Toyooka E, Kohno S, Ozawa H.. Effect of intermittentadministration of human parathyroid hormone (1-34) on the mandibular condyle of ovariectomized rats. J Bone Miner Metab. 2000; 18: 9-17.

11. Tanaka M, Ejiri S, Kohno S, Ozawa H. Region-specific bone mass changes in rat mandibular condyle following ovariectomy. J Dent Res 2000; 79: 1907-13.

12. Min HJ, Lee MJ, Kim JY et al. Alteration of BMP-4 and Runx2 expression patterns in mouse temporomandibular joint after ovariectomy. Oral Dis 2007; 13: 220-7. 
13. Rapuri PB, Gallagher JC, Balhorn KE, Ryschon KL. Alcohol intake and bone metabolism in elderly women. Am J Clin Nutr. 2000; 72: 1206-13.

14. Chakkalakal DA. Alcohol-induced bone loss and deficient bone repair. Alcohol Clin Exp Res. 2005; 29: 2077-90.

15. Sawazaki R, Lima Júnior SM, Asprino L, Moreira RW, de Moraes M. Incidence and patterns of mandibular condyle fractures. J Oral Maxillofac Surg. 2010; 68: 1252-9.

16. Yamamoto K, Kuraki M, Kurihara M, Matsusue Y, Murakami K, Horita S, et al. Maxillofacial fractures resulting from falls. J Oral Maxillofac Surg 2010; 68: 1602-7.

17. Adeyemo WL, Reuther T, Bloch W, Korkmaz Y, Fischer JH, Zöller JE, et al. Healing of onlay mandibular bone grafts covered with collagen membrane or bovine bone substitutes: a microscopical and immunohistochemical study in the sheep. Int J Oral Maxillofac Surg. 2008; 37: 651-9.

18. Berg KM, Kunins HV, Jackson JL, Nahvi S, Chaudhry A, Harris KA Jr, et al. Association between alcohol consumption and both osteoporotic fracture and bone density. Am J Med. 2008; 121: 406-18.

19. Alvisa-Negrín J, González-Reimers E, Santolaria-Fernández F, GarcíaValdecasas-Campelo E, Valls MR, Pelazas-González R, et al. Osteopenia in alcoholics: effect of alcohol abstinence. Alcohol Alcohol. 2009; 44: 468-75.

20. Broulík PD, Vondrová J, Rùzicka P, Sedlácek R, Zíma T. The effect of chronic alcohol administration on bone mineral content and bone strength in male rats. Physiol Res. 2010; 59: 599-604.

21. Marchini AMPS, Deco CP, Lodi KB, Marchini L, Santo AM, Rocha RF. Influence of chronic alcoholism and oestrogen deficiency on the variation of stoichiometry of hydroxyapatite within alveolar bone crest of rats. Arch Oral Biol. 2012; 57: 1385-94.

22. Lieber CS. Alcohol and the liver: metabolism of alcohol and its role in hepatic and extrahepatic diseases. Mt Sinai J Med. 2000; 67: 84-94.

23. Maddalozzo GF, Turner RT, Edwards $\mathrm{CH}$, Howe KS, Widrick JJ, Rosen $\mathrm{CJ}$, et al. Alcohol alters whole body composition, inhibits bone formation, and increases bone marrow adiposity in rats. Osteoporos Int. 2009; 20 : 1529-38.

24. Chakkalakal DA, Novak Jr, Fritz ED, Mollner TJ, McVicker DL, Lybarger $\mathrm{DL}$, et al. Chronic ethanol consumption results in deficient bone repair in rats. Alcohol Alcohol. 2002; 37: 13-20.

25. De Deco CP, Pereira Da Silva Marchini AM, Bárbara MA, de Vasconcellos LM, da Rocha RF, Marchini L. Negative effects of alcohol intake and estrogen deficiency combination on osseointegration in a rat model. J Oral Implantol. 2011; 37: 633-9.

26. Guyard B, Fricker J, Brigant L, Betoulle D, Apfelbaum M. Effects of ovarian steroids on energy balance in rats fed a highly palatable diet. Metabolism. 1991; 40: 529-33.

27. Kimura M, Irahara M, Yasui T, Saito S, Tezuka M, Yamano S, et al. The obesity in bilateral ovariectomized rats is related to a decrease in the expression of leptin receptors in the brain. Biochem Biophys Res Commun. 2002; 290: 1349-53.

28. Tanaka M, Ejiri S, Nakajima M, Kohno S, Ozawa H. Changes of cancellous bone mass in rat mandibular condyle following ovariectomy. Bone. 1999; 25: 339-47.

29. Ganss B, Kim RH, Sodek J. Bone sialoprotein. Crit Rev Oral Biol Med. 1999; 10: 79-98.

30. Gallagher JC. Advances in bone biology and new treatments for bone loss. Maturitas. 2008; 60: 65-9.

31. Miheller P, Muzes G, Rácz K, Blázovits A, Lakatos P, Herszényi L, et al. Changes of OPG and RANKL concentrations in Crohn's disease after infliximab therapy. Inflamm Bowel Dis. 2007; 13: 1379-84.

32. Stolina M, Kostenuik PJ, Dougall WC, Fitzpatrick LA, Zack DJ. RANKL inhibition: from mice to men (and women). Adv Exp Med Biol. 2007; 602: 143-50.

33. Valverde P, Zhang J, Fix A et al. Overexpression of bone sialoprotein leads to an uncoupling of bone formation and bone resorption in mice. J Bone Miner Res 2008; 23: 1775-88.
34. Malaval L, Wade-Guéye NM, Boudiffa M, Zhu J, Ma W, Tu Q, et al. Bone sialoprotein plays a functional role in bone formation and osteoclastogenesis. J Exp Med. 2008; 205: 1145-53.

35. Ishigaki R, Takagi M, Igarashi M, Ito K. Gene expression and immunohistochemical localization of osteonectin in association with early bone formation in the developing mandible. Histochem J. 2002; 34: 57-66.

36. Carda C, Silvestrini G, Gomez De Ferraris ME, Peydró A, Bonucci E. Osteoprotegerin (OPG) and RANKL expression and distribution in developing human craniomandibular joint. Tissue Cell. 2005; 37: 247-55.

37. Luvizuto ER, Queiroz TP, Dias SM, Okamoto T, Dornelles RC, Garcia IR $\mathrm{Jr}$, et al. Histomorphometric analysis and immunolocalization of RANKL and OPG during the alveolar healing process in female ovariectomized rats treated with oestrogen or raloxifene. Arch Oral Biol. 2010; 55: 52-9.

38. Camelo Jr JS, Martins AR, Rosa E, Ramos SG, Hehre D, Bancalari E, et al. Angiotensin II type 1 receptor blockade partially attenuates hypoxiainduced pulmonary hypertension in newborn piglets: relationship with the nitrergic system. Braz J Med Biol Res 2012; 45: 163-71.

39. Tera Tde M, Nascimento RD, Prado RF et al. Immunolocalization of markers for bone formation during guided bone regeneration in osteopenic rats. J Appl Oral Sci. 2014; 22: 541-53. 